\title{
Instillation frei von Katheter
}

Die Lokaltherapie der Blase mittels eines speziellen Adapters ist sicher und für Patienten komfortabler als die übliche Kathetertherapie. Intravesikale Instillationen werden mittlerweile auch von den Fachgesellschaften empfohlen.

Entgegen der landläufigen Meinung ist Urin per se nicht steril, eine asymptomatische Bakteriurie lässt sich bei jungen Frauen in 1-3\% und bei postmenopausalen Frauen in 13,6 bis $22,4 \%$ nachweisen, erinnert ein Expertenbrief der Schweizerischen Gesellschaft für Gynäkologie und Geburtshilfe (SGGG) [1]. Allerdings, so die Experten, ist daraus nicht zu schliessen, dass ein Eingreifen erforderlich ist: Asymptomatische Bakteriurien sollen in der Praxis bei nicht-schwangeren Frauen weder routinemässig gescreent, noch behandelt werden (falls sie als Zufallsbefund entdeckt werden), sondern ausschliesslich vor urogynäkologischen Eingriffen.

\section{Senkung der HWI-Rezidivhäufig- keit}

Von akuten Harnwegsinfektionen (HWI) wiederum ist rund die Hälfte aller Frauen betroffen, wobei bei mindestens einem Viertel ein Rezidiv auftritt; 1-3\% der unkomplizierten HWI gehen unbehandelt in eine Pyelonephritis über. Zur Senkung der Rezidivhäufigkeit, aber auch zur Behandlung einer akuten Phase des $\mathrm{HWI}$, sind laut SGGG neben D-Mannose, lokaler Östrogenisierung (bei postmenopausalen Frauen) und einem Extrakt aus uropathogenen E.coli-Stämmen auch intravesikale Installationen aus Hyaluronsäure und Chondroitinsulfat empfohlen, welche die Glykosaminoglykanschicht der Blase aufbauen; einige Krankenversicherer übernehmen hier die Kosten.

Die intravesikale Verabreichung von Medikamenten kommt außerdem bei Blasenkarzinom zur Senkung der lokalen Rezidivrate, bei interstitieller Zystitis beziehungsweise BPS („bladder pain syndrome"), bei chronisch-bakterieller Blaseninfektion und nach Strahlentherapie des Beckens zum Einsatz. In den meisten Fällen wird ein Katheter zurVerabreichung benutzt, doch bringt jede Katheterisierung die bekannten Risiken mikro- oder makrovaskulärer Läsionen der Blasenschleimhaut und damit auch Schmerzen, Blutungen und ein höheres Infektionsrisiko mit sich, warnen die Autoren einer ungarischen Studie [2].

\section{Adapter an 270 Patienten getestet}

Sie untersuchten einen von ihnen entwickelten Adapter, der sowohl auf LuerLock als auch auf Luer-Slip Spritzen aufgesetzt werden kann. Nach Desinfektion der Harnröhrenöffnung und der Umgebung wird die Spitze des Adapters in die Öffnung eingeführt und die Spritze mit leichtem Druck appliziert, um vollen Kontakt mit der periurethralen Mukosa zu erlauben. Danach wird die Wirkstofflösung durch die Harnröhre in die Blase eingebracht. Dieser Vorgang erlaubt also eine retrograde Wirkstoffinjektion in die Blase durch die Urethra ohne Katheterisierung.

270 Patienten (243 Frauen, 27 Männer) mit interstitieller Zystitis/PBS erhielten insgesamt 1520 Injektionen. Die Ergebnisse bestätigen zunächst die Effektivität dieser Vorgehensweise: Bei lediglich fünf der 243 Patientinnen (2\%) war der Einsatz des SpritzenAdapters aufgrund einer tief sitzenden Harnröhrenöffnung beziehungsweise einer vernarbten Vaginalöffnung nicht erfolgreich; die Erfolgsrate bei den Patientinnen lag damit bei $98 \%$. Von den männlichen Patienten konnten $100 \%$ erfolgreich und ohne Schwierigkeiten behandelt werden.

Auch Sicherheit und Verträglichkeit wurden untersucht. Bakterielle Infektionen aufgrund der Instillation wurden nicht beobachtet; Schmerzen, langanhaltendes Brennen oder andere Komplikationen wurden nicht angegeben. Die Akzeptanz durch die Patientinnen und Patienten lag bei $100 \%$ : Alle Teilnehmerinnen und Teilnehmer bevorzugten die katheterlose Methode zur konventionellen Katheterisierung; keiner der Probanden zog nachfolgend „eine Katheterisierung auch nur in Erwägung", schließen die Autoren.

\section{Literatur \\ 1. Betschart C, Albrich W, Brandner S, Faltin D, Kuhn A, Surbek D, Geissbühler V (2018) Aku- te und rezidivierende Harnwegsinfektionen. Expertenbrief No 58. Schweizerische Gesell- schaft für Gynäkologie und Geburtshilfe \\ 2. Lovasz S (2019) Minimally invasive device for intravesical instillation by urological syringe adapter (MID-ii U.S.A.) for catheter-free ins- tillation therapy of the bladder in interstitial cystitis/bladder pain syndrome. Int J Urol 26(Suppl 1):57-60. https://doi.org/10.1111/ iju.13976}

Autorin: Dr. Lydia Unger-Hunt

Urol. Prax. 2019 · 21:166

https://doi.org/10.1007/s41973-

019-00081-y

(c) Springer-Verlag GmbH Austria, ein Teil von Springer Nature 2019 